\title{
ALCAPA: The AI Capone of coronary artery anomalies
}

\author{
Farzanah Ismail, FCRad (D) SA, MMed Rad (D) \\ Department of Radiology, Steve Biko Academic Hospital and University of Pretoria
}

Corresponding author: F Ismail (drfismail@yahoo.com)

\begin{abstract}
Anomalous left coronary artery originating from the pulmonary artery (ALCAPA) is a rare coronary artery anomaly that presents with myocardial ischaemia or infarction and/or cardiac failure in infants. It is associated with a mortality rate of $90 \%$ within the first year of life. Surgical correction to re-establish a two-coronary artery perfusion system is the treatment of choice, once patients are medically stable.
\end{abstract}

S Afr J Rad 2012;16(3):100-101. DOI:10.7196/SAJR.633

\section{Clinical presentation}

A 12-month-old infant presented with tachycardia and respiratory distress. On clinical evaluation, the child was in cardiac failure. There were no clinical features of infection. Haematological studies were normal. The electrocardiogram (ECG) demonstrated septal ischaemic changes. The chest radiograph (Fig. 1) demonstrated gross cardiomegaly involving mainly the ventricles and right atrium. There was peri-hilar alveolar opacification and a left pleural effusion. There was a positive silhouette sign at the right cardiac border, indicating right middle lobe consolidation. These findings were due to pulmonary oedema or infection. The differential diagnosis at this stage was dilated

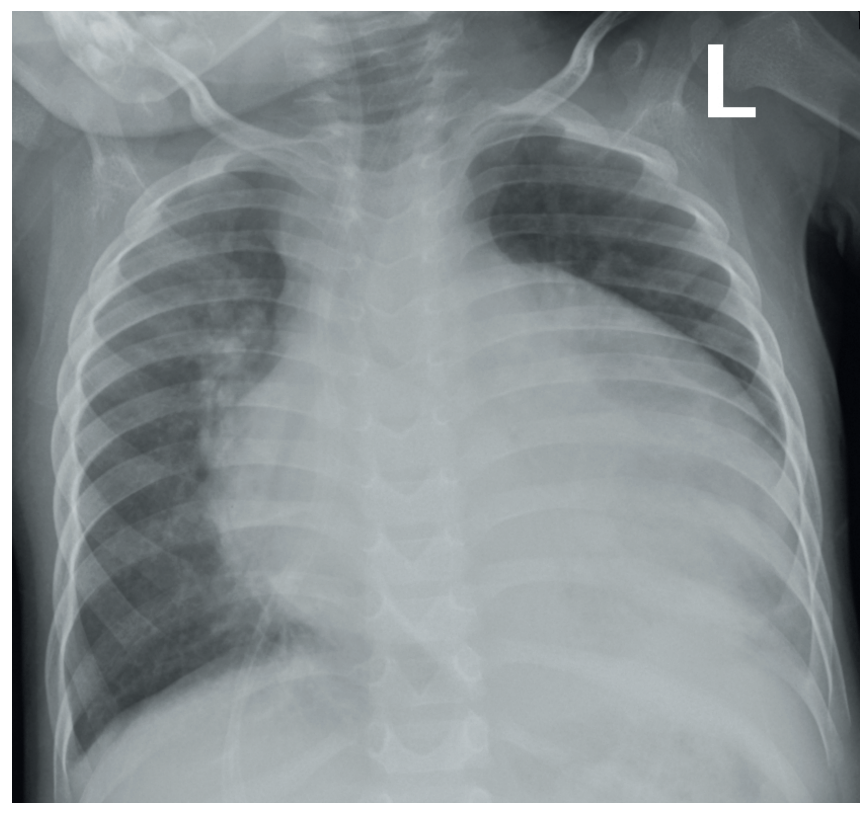

Fig. 1. Antero-posterior radiograph of the chest demonstrates massive cardiomegaly, involving mainly the ventricles and right atrium. Peri-hilar alveolar opacification and left pleural effusion as well as right middle lobe consolidation are present. cardiomyopathy, viral myocarditis, mitral valve disease or anomalous left coronary artery originating from the pulmonary artery (ALCAPA). Echocardiography excluded cardiomyopathy and mitral valve disease. Coronary angiography confirmed ALCAPA (Figs 2 and 3). The patient was evaluated for surgery.

\section{Discussion}

Anomalous left coronary artery originating from the pulmonary artery (ALCAPA) is a rare coronary artery anomaly that affects 1 in 300000 live births. ${ }^{1}$ It was first described in 1886, but Bland, Garland and White described in 1933 the landmark case describing the clinical features linked with ALCAPA; hence it is also known as Bland-White-Garland syndrome. ${ }^{2,3}$

There are two types of ALCAPA syndrome: the adult and the infant type. The infant type presents with myocardial ischaemia or infarction and/or cardiac failure, and has a mortality rate of $90 \%$ within the first year of life. ${ }^{1}$ Infants may also present with paroxysms of irritability which correlate with episodes of angina. ${ }^{4}$ ALCAPA can occur in isolation or in conjunction with other lesions such as atrial septal defect and ventricular septal defect. ${ }^{4}$

Embryologically, the anomaly arises from either abnormal septation of the aorta and the pulmonary artery, or from persistence of aortic buds that form the coronary arteries. ${ }^{2}$ In the neonatal period, the baby is asymptomatic as there is anterograde flow of desaturated blood from the pulmonary artery to the left coronary artery. As pulmonary arterial pressure drops, the combination of low flow and desaturated blood causes myocardial ischaemia, especially during exertion. Collateral vessels develop between the right and left coronary arteries. Further decreases in pulmonary arterial pressure result in reversal of flow, as the left coronary artery drains from the right coronary artery, through collaterals, into the pulmonary artery. This is known as myocardial steal; hence the nickname of $\mathrm{Al}$ Capone of coronary vessels. ${ }^{2,4}$

The steal phenomenon causes ischaemia or infarction of the anterolateral LV wall. The electrocardiogram findings include a QR pattern followed by inverted $\mathrm{T}$ waves seen on leads I and aVL. The left ventricular surface leads $\left(V_{5}-V_{6}\right)$ may also show deep $Q$ waves and exhibit elevated ST segments and inverted T waves. ${ }^{2}$ Patients who survive to adulthood have a good collateral network with large-calibre left and right coronary arteries. They may present with dysrrythmias or sudden death.

Chest radiography may demonstrate left ventricular hypertrophy and dilation secondary to chronic myocardial ischaemia. ${ }^{1}$ The main differential diagnosis at this stage is cardiomyopathy, which is excluded on echocardiography. Echocardiography may also demonstrate the anomalous left coronary artery with Doppler flow confirming the reversal of flow. ${ }^{2}$ Coronary artery angiography will additionally 


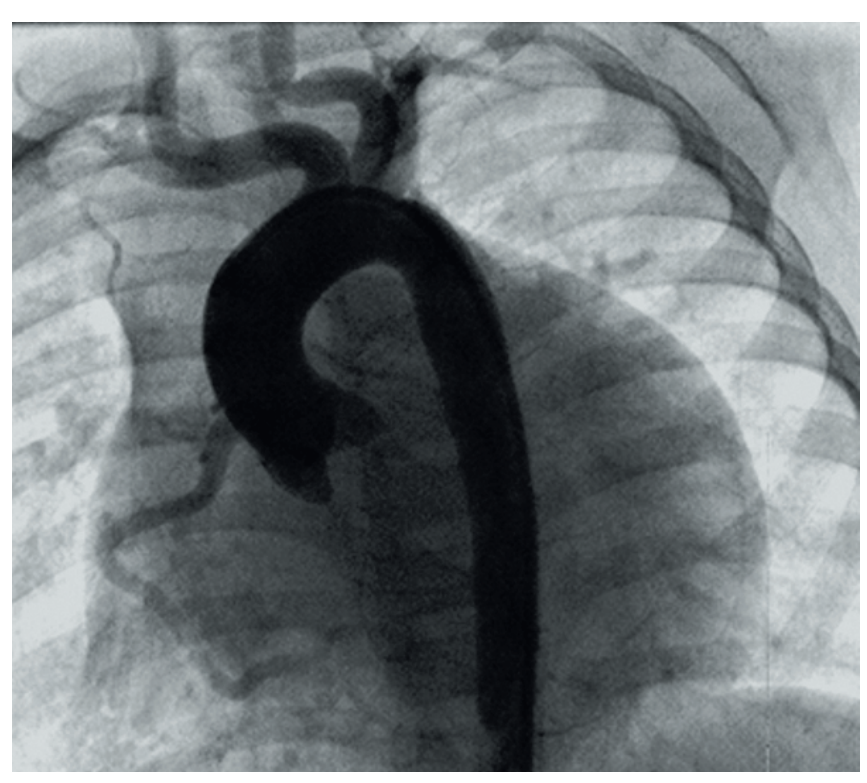

Fig. 2. Antero-posterior aortic angiogram with catheter in the ascending aorta. Only the right coronary artery fills with contrast. The left coronary artery is not visualised. Cardiomegaly is present. Feint contrast within the pulmonary arteries is also visible.

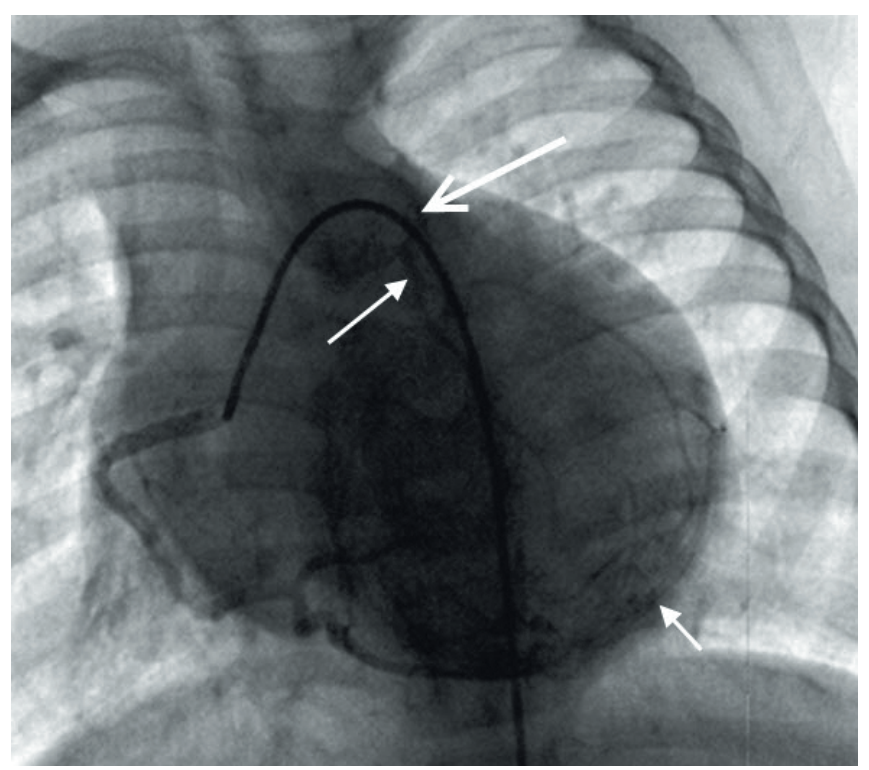

Fig. 3. Selective catheterisation of the right coronary artery. Multiple collateral vessels are present (smallest white arrow) which drain into the left coronary artery (arrow to the left of catheter) and into the main pulmonary artery (largest white arrow).

demonstrate dilated tortuous vessels and reversal of flow into the pulmonary artery on delayed images. CT coronary angiography has the benefit of being non-invasive and will also demonstrate the origin of the left coronary artery from the pulmonary artery. Anomalous origin of the other coronary vessels may also be identified. The retrograde flow from the LCA to the pulmonary artery is well depicted on MRI and is seen as a jet of blood flowing into the pulmonary artery. ${ }^{1}$
Medical management involves treatment of cardiac failure. ${ }^{2}$ Surgical correction to re-establish a two-coronary artery perfusion system is the treatment of choice, once patients are medically stable. The surgery is performed preferably in the neonatal period as it is in this age group that there is some improvement of left ventricular function. ${ }^{1}$

\section{Conclusion}

The steal phenomenon caused by ALCAPA causes significant left ventricular myocardial compromise and is associated with a high morbidity in infants. An infant who presents with LV enlargement, ischaemic ECG changes and/or features of cardiac failure should be considered to have ALCAPA until proven otherwise.

1. Pena E, Nguyen ET, Merchant N, Dennie C. ALCAPA syndrome: Not just a pediatric disease. Radiographics 2009;29:553-565.

2. Lardhi AA. Anomalous origin of left coronary artery from pulmonary artery: A rare cause of myocardial infarction in children. Journal of Family Community Medicine 2010;17:113-116.

3. Cowles RA, Berdon WE. Bland-White-Garland syndrome of anomalous left coronary artery arising from the pulmonary artery: A historical review. Pediatr Radiol 2007;37:890-895.

4. Brotherton H, Phillip RK. Anomalous left coronary artery from pulmonary artery (ALCAPA) in infants: a 5 year review in a defined birth cohort. Eur J Pediatr 2008;167:43-46. 